\title{
Antibiotic therapy and fat digestion and absorption in cystic fibrosis
}

\author{
Aleksandra Lisowska ${ }^{1 凶}$, Andrzej Pogorzelski², Grzegorz Oracz ${ }^{3}$, Wojciech Skorupa ${ }^{4}$, Szczepan \\ Cofta5, Jarosław Szydłowski ${ }^{6}$, Jerzy Socha ${ }^{3}$ and Jarosław Walkowiak ${ }^{1,7}$ \\ $1^{1 \text { st }}$ Chair of Pediatrics, Department of Pediatric Gastroenterology \& Metabolism, Poznań University of Medical Sciences, Poznań, Poland; ${ }^{2}$ De- \\ partment of Bronchology \& Cystic Fibrosis, National Institute for Tuberculosis \& Lung Diseases, Pediatric Branch, Rabka, Poland; ${ }^{3}$ Department of \\ Pediatric Gastroenterology, Hepatology \& Immunology, Child Memorial Health Institute, Warszawa, Poland; ${ }^{4}$ st Clinic of Lung Diseases, National \\ Tuberculosis and Lung Diseases Research Institute, Warszawa, Poland; ${ }^{5}$ Department of Respiratory Medicine, ${ }^{6}$ Department of Pediatric Otorhi- \\ nolaryngology, Poznań University of Medical Sciences, Poznań, Poland; ' Department of Dietetics, Chair of Human Nutrition \& Hygiene, Poznań \\ University of Life Sciences, Poznań, Poland
}

Antibiotic therapy in the cystic fibrosis (CF) mouse model has been shown to result in reduced bacterial load of the intestine and significant body mass gain. The effect was suggested to be linked to the improvement of intestinal digestion and absorption. Therefore, we aimed to assess the influence of routinely applied antibiotic therapy in CF patients on fat assimilation. Twenty-four CF patients aged 6 to 30 years entered the study. Inclusion criteria comprised confirmed exocrine pancreatic insufficiency and bronchopulmonary exacerbation demanding antibiotic therapy. Exclusion criteria comprised: antibiotic therapy six weeks prior to the test, liver cirrhosis, diabetes mellitus, oxygen dependency, the use of systemic corticosteroids. In all enrolled CF subjects, ${ }^{13} \mathrm{C}$-labelled mixed triglyceride breath test $\left({ }^{13} \mathrm{C}\right.$ MTG-BT) was performed to assess lipid digestion and absorption, before and after antibiotic therapy. Sixteen subjects were treated intravenously with ceftazidime and amikacin, eight patients orally with ciprofloxacin. Cumulative percentage dose recovery (CPDR) was considered to reflect digestion and absorption of lipids. The values are expressed as means (medians). The values of CPDR before and after antibiotic therapy did not differ in the whole studied group [4.6(3.3) \% vs. 5.7(5.3)\%, $p=0.100$ ] as well as in the subgroup receiving them intravenously [4.6(3.2)\% vs. 5.7(5.3) $\%, p=0.327]$ or in that with oral drug administration [4.6(3.4) \% vs. 5.7(5.4) \%, $p=0.167]$. In conclusion, antibiotic therapy applied routinely in the course of pulmonary exacerbation in CF patients does not seem to result in an improvement of fat digestion and absorption.

Keywords: cystic fibrosis, antibiotic therapy, malabsorption, stable isotope breath test

Received: 10 December, 2010; revised: 13 May, 2011; accepted: 05 July, 2011; available on-line: 07 July, 2011

\section{INTRODUCTION}

Cystic fibrosis (CF) is an inflammatory and destructive disease with differentiated clinical expression. Bronchopulmonary disease is the major clinical manifestation which frequently demands antibiotic therapy. Available data suggests that such treatment applied in CF patients improves not only respiratory function but also nutritional status, which has been related to increased energy intake and decreased energy expenditure (Vic et al., 1997; Castro et al., 2002; Hankard et al., 2002; Béghin et al.,
2003). Interestingly, antibiotic therapy in the CF mouse model resulted in the reduction of bacterial load of the small intestine, decreased intensity of intestinal inflammation and significant body mass gain. The effect was suggested to be linked to the improvement of intestinal digestion and absorption (Norkina et al., 2004a; 2004b). Therefore, we aimed in the present study to assess the influence of routinely applied antibiotic therapy in CF patients on fat digestion and absorption.

\section{MATERIAL AND METHODS}

The study comprised 24 CF patients (14 females and 10 males) (Table 1). Diagnosis of CF was based on history, clinical manifestation and increased sweat chloride concentrations and confirmed by the CFTR gene analysis. The genotypes of the studied patients were as follows: F508del/F508del $(n=11), \quad$ F508del/CFTRdele2,3 (21 kb) $(\mathrm{n}=2)$, F508del/R553X $(\mathrm{n}=1), \quad$ F508del $/ 2143 \mathrm{del} T$ $(\mathrm{n}=1), \quad$ CFTRdele2,3 (21 kb)/CFTRdele2,3 (21 kb) $(\mathrm{n}=1), 1717-1 \mathrm{G}-\mathrm{A} / \mathrm{N} 1303 \mathrm{~K}(\mathrm{n}=1), \quad$ F508del $/ 2143 \mathrm{delT}$ $(\mathrm{n}=1), \quad$ F508del $/ 2183 \mathrm{AA}-\mathrm{G} \quad(\mathrm{n}=1), \quad$ F508del/W1282X $(\mathrm{n}=1), 1717-1 \mathrm{G}-\mathrm{A} / \mathrm{N} 1303 \mathrm{~K}(\mathrm{n}=1), \mathrm{F} 508 \mathrm{del} /$ unknown mutation $(\mathrm{n}=2)$, unknown mutation/unknown mutation $(\mathrm{n}=1)$.

Inclusion criteria for subjects comprised the willingness to participate in the study and exocrine pancreatic insufficiency (fecal elastase-1 concentration $<100 \mu \mathrm{g} / \mathrm{g}$ and the presence of steatorrhea) (Walkowiak, 2004; Walkowiak et al., 2005). Exclusion criteria comprised: intravenous and oral antibiotic therapy six weeks prior to the test, liver cirrhosis, diabetes mellitus, oxygen dependency, the use of systemic corticosteroids.

In all enrolled $\mathrm{CF}$ subjects, ${ }^{13} \mathrm{C}$-labelled mixed triglyceride breath test ( ${ }^{13} \mathrm{C}$ MTG-BT) was performed to assess lipid digestion and absorption, before and after antibiotic therapy (on the day preceding antibiotic therapy and on the last day of antibiotic administration). Sixteen patients were treated intravenously with ceftazidime and amikacin, in respective doses: $150-250 \mathrm{mg} / \mathrm{kg}$ per $24 \mathrm{~h}$ and $20-35 \mathrm{mg} / \mathrm{kg}$ per $24 \mathrm{~h}$, the remaining eight subjects were given ciprofloxacin orally in a dose of $35-50 \mathrm{mg} /$

e-mail: alisow1@wp.pl

Abbreviations: CF, cystic fibrosis; CFTR, cystic fibrosistransmembrane conductance regulating protein; ${ }^{13} \mathrm{C}$ MTG-BT, ${ }^{13} \mathrm{C}$-labelled mixed triglyceride breath test; CPDR, cumulative percentage dose recovery; CFA, coefficient of fat absorption 
Table 1. Basic epidemiological and clinical data of cystic fibrosis patients $(n=24)$

\begin{tabular}{lll}
\hline Parameter & Range & Mean (median) \\
\hline Age (years) & $10-32$ & $19.8(18)$ \\
Body mass (Z-score) & $-2.42-1.02$ & $-0.89(-0.94)$ \\
FEV1a (\%) & $55-105$ & $78(77)$ \\
Fecal elastase-1 $(\mu \mathrm{g} / \mathrm{g})$ & BDL-88 & $12(7)$ \\
AlATc (U/l) & $8-52$ & $24.4(24)$ \\
GGTPd $(\mathrm{U} / \mathrm{l})$ & $6-98$ & $18.9(14)$
\end{tabular}

aforced expiratory volume in $1 \mathrm{~s}$; bbelow detection limit; calanine transaminase (EC 2.6.1.2); ${ }^{\mathrm{d}} \mathrm{\gamma}$-glutamyltransferase (EC 2.3.2.2)

$\mathrm{kg}$ per $24 \mathrm{~h}$. The antibiotics were administered for 14 days.

${ }^{13} \mathrm{C}$ MTG-BT was performed after overnight fast. Each of the studied subjects received $150 \mathrm{mg}$ of ${ }^{13} \mathrm{C}$ mixed triglyceride with $0.25 \mathrm{~g}$ butter per $\mathrm{kg}$ body mass mixed on a slice of bread. Breath samples were collected at baseline (fasting) and at 30, 60, 90, 120, 150, 180, 210, 240, 270, 300, 330 and 360 minutes after test meal ingestion. The samples were analyzed with an IRIS ${ }^{13} \mathrm{C}$ Analyser System (Wagner, Bremen, Germany). Cumulative percentage dose recovery (CPDR) was considered to reflect digestion and absorption of lipids.

Values are expressed as ranges, means and medians. The statistical significance of differences in CPDR before and after antibiotic therapy was determined with the use of Wilcoxon-rank test. The level of significance was set at $p<0.05$. Statistical analysis was performed using STATISTICA 8.0. (StatSoft Inc. 2008).

The protocol of the investigation was approved by the Bioethical Committee of Poznań University of Medical Sciences, Poland.

\section{RESULTS}

The values of CPDR before and after antibiotic therapy did not differ in the whole studied group or in either of the subgroups differing in the mode of drug administration (Table 2).

\section{DISCUSSION}

No significant influence of the antibiotic treatment applied on lipid digestion and absorption was observed in

Table 2. Lipid digestion and absorption in cystic fibrosis (CF) patients undergoing antibiotic therapy based upon cumulative ${ }^{13} \mathrm{C}$ dose recovery (CPDR)

\begin{tabular}{llccc}
\hline \multirow{2}{*}{ Studied group ( $\mathrm{n}$ ) } & \multicolumn{2}{l}{} & \multirow{2}{*}{ SPDR } \\
\cline { 2 - 4 } & Parameter & Before & After & \\
\hline CF (24) & Mean (median) & $4.6(3.4)$ & $5.8(5.3)$ & $p=0.100$ \\
& Range & $0-20.2$ & $0.8-12.8$ & \\
CF-IV* (16) & Mean (median) & $4.6(3.2)$ & $5.7(5.3)$ & $p=0.327$ \\
& Range & $0-20.2$ & $1.8-11.4$ & \\
CF-PO** (8) & Mean (median) & $4.7(3.4)$ & $5.7(5.4)$ & $p=0.167$ \\
& Range & $0-9.5$ & $0-12.8$ & \\
\hline
\end{tabular}

${ }^{*} \mathrm{CF}-\mathrm{IV}$, subjects receiving antibiotics intravenously; ${ }^{* *} \mathrm{CF}-\mathrm{PO}$, subjects receiving antibiotics orally the present study. To the best of our knowledge this is the first study assessing such a relationship in humans in a reliable way.

Reilly et al. (1999) made an attempt to assess the effect of an acute respiratory exacerbation on energy balance. The exacerbation was associated with a significant reduction in energy intake. A trend towards lower total energy expenditure was observed. No statistical differences in fat absorption and resting energy expenditure as well as body mass and composition were documented. However, the power of the study was rather low, as only 14 children were studied. The data on the effectiveness of fat digestion and absorption (coefficient of fat absorption - CFA) were available for ten of them. CFA was better during exacerbation in seven subjects and worse in one patient. The authors attributed some CFA changes to a better compliance with the pancreatic enzyme replacement therapy under supervision. However, they finally concluded that the changes were negligible. With the methodology applied in their study it is difficult to determine the reliability of the findings. Fat intake was assessed during exacerbation for 6-7 days and for one weekend and two week days during the stable period. Fecal fat output was calculated from a three-day stool collection made during each period. Nevertheless, the relationship between the timing of food intake and stool collection as well as between stable period, antibiotic treatment and exacerbation was not precised. According to the authors' discussion they aimed to assess the difference in CFA between well-being and exacerbation. In contrast to their study, we assessed the effect of both oral and intravenous antibiotic therapies. In addition, we determined fat assimilation on fixed days before and after antibiotic treatment (reflecting in a reliable way appropriate time points) to assess its potential influence on the efficacy of digestion and absorption.

There is a strong association of bacterial infection and inflammation in CF, at least in the airways. Accumulating evidence indicates that susceptibility to inflammation may be inherent to the tissue involved even in the absence of specific pathogenic microbial colonization (Muhlebach et al., 1999). The CFTR-null mouse model does not express CFTR and is not expected to have inherent inflammation due to protein misfolding. The observed inflammation in the CF mouse intestine most likely occurs as a result of altered luminal environment with subsequent bacterial overgrowth (Norkina et al., 2004a; 2004b). The body weight of CF mice at the end of 3 -week antibiotic treatment (ciprofloxacin and metronidazole) was significantly increased compared to untreated CF mice and not significantly different from that of wild-type animals. The antibiotic treatment had no effect on the body mass of control wild-type animals (Norkina et al., 2004b). The obtained results suggest that antibiotic therapy may have a significant impact on digestion and absorption in CF patients, thereby influencing the energy balance during treatment of pulmonary exacerbations. The doses used in the animal model are not easy to be translated into a human study. Moreover, ciprofloxacin doses used in bronchopulmonary exacerbation in CF are well established. Therefore, we applied its typical 
doses. Although intravenous therapy in such cases is more commonly used, we also assessed the effects of oral antibiotic administration on lipid assimilation. The observed changes did not reach the level of significance. However, a tendency towards an improvement of fat absorption was noted.

In conclusion, routinely applied antibiotic therapy in the course of pulmonary exacerbation in CF patients does not seem to result in an improvement of fat digestion and absorption.

\section{Acknowledgements}

Supported by the Ministry of Science and Higher Education (grant No. N 406054 31).

CFTR gene analysis was performed in the Department of Human Genetics, Polish Academy of Sciences, Poznań, Poland (Michał Witt, Ewa Rutkiewicz) and the Department of Clinical Genetics, Mother and Child Institute, Warszawa, Poland (Agnieszka SobczyńskaTomaszewska, Jerzy Bal).

The authors declare no conflict of interest.

\section{REFERENCES}

Béghin L, Gottrand F, Michaud L, Loeuille GA, Wizla-Derambure N, Sardet A, Guimber D, Deschildre A, Turck D (2003) Impact of intravenous antibiotic therapy on total daily energy expenditure and physical activity in cystic fibrosis children with Pseudomonas aeruginosa pulmonary exacerbation. Pediatr Res 54: 756-761.

Castro M, Diamanti A, Gambarara M, Bella S, Lucidi V, Papadatou B, Ferretti F, Rosati P, Rupi E (2002) Resting energy expenditure in young patients with cystic fibrosis receiving antibiotic therapy for acute respiratory exacerbations. Clin Nutr 21: 141-144.

Hankard R, Munck A, Navarro J (2002) Nutrition and growth in cystic fibrosis. Horm Res 58 (Suppl 1): 16-20.

Muhlebach MS, Stewart PW, Leigh MW, Noah TL (1999) Quantitation of inflammatory responses to bacteria in young cystic fibrosis and control patients. Am J Respir Crit Care Med 160: 186-191.

Norkina O, Burnett DG, De Lisle RC (2004a) Bacterial overgrowth in cystic fibrosis transmembrane conductance regulator null mouse small intestine. Infect Immun 72: 6040-6049.

Norkina O, Kaur S, Ziemer D, De Lisle RC (2004b) Inflammation of the cystic fibrosis mouse small intestine. Am J Physiol Gastrointest Liver Physiol 286: G1032-G1041.

Reilly JJ, Evans TJ, Wilkinson J, Paton JY (1999) Adequacy of clinical formulae for estimation of energy requirements in children with cystic fibrosis. Arch Dis Child 81: 120-124.

Vic P, Ategbo S, Gottrand F, Launay V, Loeuille GA, Elian JC, Druon D, Farriaux JP, Turck D (1997) Nutritional impact of antipseudomonas intravenous antibiotic courses in cystic fibrosis. Arch Dis Child 76: 437-440.

Walkowiak J (2004) Assessment of maldigestion in cystic fibrosis. J Pediatr 145: 285-287.

Walkowiak J, Nousia-Arvanitakis S, Henker J, Stern M, Sinaasappel M, Dodge JA (2005) Indirect pancreatic function tests in children. J Pediatr Gastroenterol Nutr 40: 107-114. 